\title{
Evidence for crossed Andreev reflection in superconductor-ferromagnet hybrid structures
}

\author{
D. Beckmann* and H. B. Weber \\ Forschungszentrum Karlsruhe, Institut für Nanotechnologie, P.O. Box 3640, D-76021 Karlsruhe, Germany
}

H. v. Löhneysen

Forschungszentrum Karlsruhe, Institut für Festkörperphysik, P.O. Box 3640, D-76021 Karlsruhe, Germany, and Physikalisches Institut, Universität Karlsruhe, D-76128 Karlsruhe, Germany

(Dated: October 25, 2018)

\begin{abstract}
We have measured the non-local resistance of aluminum-iron spin-valve structures fabricated by e-beam lithography and shadow evaporation. The sample geometry consists of an aluminum bar with two or more ferromagnetic wires forming point contacts to the aluminum at varying distances from each other. In the normal state of aluminum, we observe a spin-valve signal which allows us to control the relative orientation of the magnetizations of the ferromagnetic contacts. In the superconducting state, at low temperatures and excitation voltages well below the gap, we observe a spin-dependent non-local resistance which decays on a smaller length scale than the normal-state spin-valve signal. The sign, magnitude and decay length of this signal is consistent with predictions made for crossed Andreev reflection (CAR).
\end{abstract}

Singlet superconductivity and ferromagnetism are competing long-range orders which favor a different alignment of electron spins, antiparallel and parallel, respectively. Therefore, they generally exclude each other in homogenous bulk materials. In mesoscopic hybrid structures, the interplay of superconductivity and ferromagnetism leads to rich novel physics. Recent experimental studies on equilibrium properties of superconductorferromagnet (SF) interfaces have shown that the local density of states (LDOS) on the superconducting side is strongly affected by the pair breaking effect of the ferromagnetic exchange field [1], and on the ferromagnetic side an oscillatory behavior of the LDOS due to the exchange splitting of the spin sub-bands has been observed 2]. Transport properties of SF point contacts [3, 4, 5] and FSF planar junctions [6] show a suppression of spin-polarized current injection into the superconductor, and in SFS Josephson junctions [7, 8, 9], a $\pi$ state with a spontaneous equilibrium Josephson current is observed. In this work, we report on spin-dependent transport properties of the superconducting condensate on length scales comparable to the coherence length. We have combined ferromagnet-superconductor point contacts with a non-local spin-valve geometry, and find evidence for crossed Andreev reflection (CAR), i.e. the splitting of a Cooper pair into two spatially separated leads.

Figure 1 shows an SEM image of one of our samples, together with a schematic view of the experiment. The samples were fabricated by e-beam lithography and shadow evaporation techniques. First, $20 \mathrm{~nm}$ of iron is evaporated onto an oxidized silicon substrate to form a series of wires (vertical), with varying width (50-120 nm) and a small tip at the end. Their elongated shape creates a magnetic shape anisotropy which confines their magne-

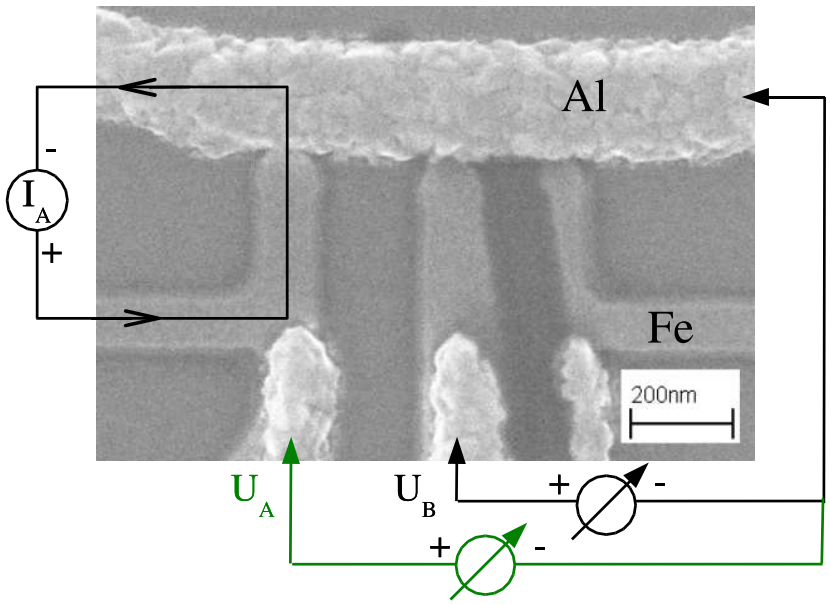

FIG. 1: (Color online) SEM image of sample T2, and experimental scheme. Three vertical iron wires are connected by point contacts to a horizontal aluminum bar. The outer two iron wires have additional voltage probes. An example of the current injection $\left(I_{\mathrm{A}}\right)$ and both local $\left(U_{\mathrm{A}}\right)$ and non-local $\left(U_{\mathrm{B}}\right)$ voltage detection scheme for one pair of contacts is also shown.

tizations to be aligned along the wires, pointing either upwards or downwards, with different coercive fields due to the width variation. In a second evaporation step under a different angle, and without breaking the vacuum, an aluminum bar of $80 \mathrm{~nm}$ height and $250 \mathrm{~nm}$ width (horizontal), is created. It slightly touches the iron wires, forming metallic contacts of about $20 \times 50 \mathrm{~nm}$, much smaller than the dirty-limit coherence length $\xi \approx 200-300 \mathrm{~nm}$ of the aluminum. This is crucial to avoid a suppression of superconductivity by the proximity effect. A current $I_{\mathrm{A}}$ is injected through one of the contacts into the superconductor, and a voltage $U_{\mathrm{B}}$ is detected by a second contact 
outside the current path, defining the non-local resistance $R=U_{\mathrm{B}} / I_{\mathrm{A}}$. Samples of two different layouts were investigated. Layout $\mathrm{T}$ (shown) has three iron wires and additional voltage probes for detection of the local resistance $U_{\mathrm{A}} / I_{\mathrm{A}}$. Layout $\mathrm{S}$ has six iron wires over a larger range of distances, and no additional voltage probes. For each sample, different combinations of contacts have been used as injector-detector pair in order to study the dependence of $R$ on the distance $d$ between the two contacts. Here, we show data from one sample of each layout, T2 and S5. The samples were mounted into a shielded box thermally anchored to the mixing chamber of a dilution refrigerator. The measurement lines were fed through a series of filters to eliminate $\mathrm{RF}$ and microwave radiation from the shielded box. Resistance was measured with a lowfrequency AC resistance bridge, with an RMS excitation amplitude $I_{\text {exc }}$. For differential resistance measurements, an additional DC bias current was applied.

For each pair of contacts, we first characterize the spinvalve behavior in the normal state. The inset of Fig. 2 shows the non-local resistance for two contacts as a function of the magnetic field $B$ applied along the direction of the ferromagnetic wires at a temperature $T=1.8 \mathrm{~K}$, with an excitation amplitude $I_{\text {exc }}=1 \mu \mathrm{A}$. For each direction of the magnetic field, the data show two sharp stepwise changes of the resistance which correspond to the reversal of the magnetizations of the two contacts at their individual coercive fields, yielding each two states for parallel and antiparallel magnetization alignment. These states remain stable at zero external magnetic field. The resistance difference $\Delta R_{\mathrm{N}}$ between parallel and antiparallel alignment in the normal state is governed by the injection of non-equilibrium magnetization into aluminum, and the associated splitting of the chemical potentials for spin up and down electrons [10]. A quantitative analysis of $\Delta R_{\mathrm{N}}$ will be given below.

For each of the four magnetization states of a given contact pair, the sample is cooled through the superconducting transition at zero external magnetic field, and subsequently warmed up again. Here a very small excitation amplitude $I_{\mathrm{exc}}=50 \mathrm{nA}$ is chosen to avoid both quasiparticle injection above the superconducting gap and self-heating. At this small excitation (and correspondingly small detector voltage amplitude $U_{\mathrm{B}} \approx$ $0.5 \mathrm{nV}$ ), the limited absolute accuracy of the resistance bridge yields an artificial offset of $\approx 150 \mathrm{~m} \Omega$ to $R$. The observed data depend only on the relative magnetization alignment of the two Fe wires, and are therefore averaged over parallel and antiparallel configurations. The results for one pair of contacts is shown in Fig. 22 Above the superconducting transition temperature, the signal is constant, with the well resolved difference $\Delta R_{\mathrm{N}}$ between parallel and antiparallel alignment. At the superconducting transition at $T_{\mathrm{c}} \approx 1.15 \mathrm{~K}$, a large peak is observed, which is essentially independent of the magnetization alignment. This peak is due to charge imbalance

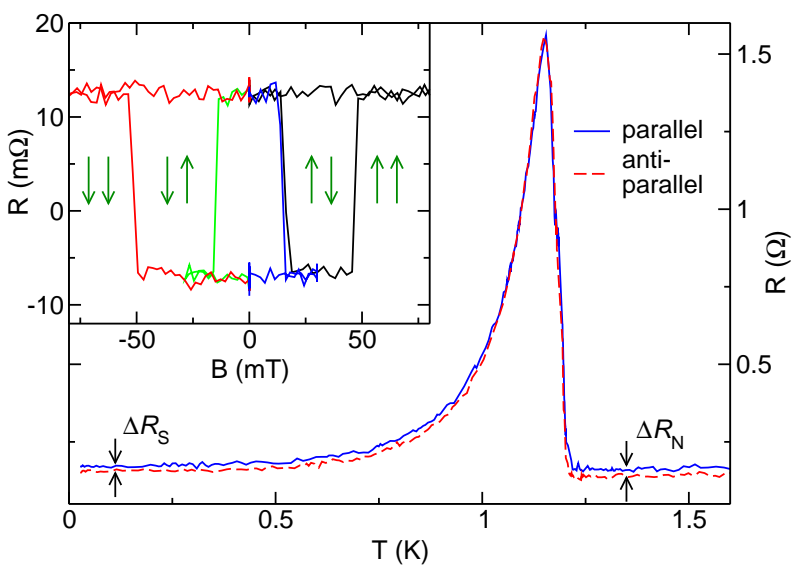

FIG. 2: (Color online) Non-local resistance $R$ as a function of the temperature $T$ at zero external magnetic field for two contacts of sample T2 at a distance $d=310 \mathrm{~nm}$. The solid and dashed line corresponds to parallel and antiparallel alignment of the injector and detector magnetizations, respectively.

Inset: Non-local resistance $R$ at $T=1.8 \mathrm{~K}$ as a function of a magnetic field $B$ applied parallel to the iron wires, for two contacts of sample S5 at a distance $d=210 \mathrm{~nm}$. The resistance jumps correspond to magnetization reversals of the ferromagnetic contacts. The arrows indicate the four different magnetization states.

created by the injection of quasiparticles above the superconducting gap 11, 12], which is still small close to $T_{\mathrm{c}}$. As the gap opens further towards lower temperatures, the charge-imbalance peak subsides, and finally, the resistance becomes constant again at $T<250 \mathrm{mK}$. In this regime, the signal for parallel alignment is still somewhat larger than for the antiparallel case. The difference $\Delta R_{\mathrm{S}}$ between the non-local resistances for parallel and antiparallel alignment in the superconducting state is the central result of this work, and we will now further clarify its physical origin.

A possible source of the spin-dependent signal in the superconducting state are quasiparticles propagating above the superconducting gap. Such a signal has been observed in the charge-imbalance region near $T_{\mathrm{c}}$ using current injection into a niobium film through a tunnel junction [13. It was found to be an order of magnitude smaller than the normal-state spin-valve signal at a temperature $0.3 \%$ below $T_{\mathrm{c}}$, and became unobservably small about $1.5 \%$ below $T_{\mathrm{c}}$. We do not observe such a signal close to $T_{\mathrm{c}}$, presumably because it is buried in the steep spin-independent increase caused by charge imbalance.

To check further whether $\Delta R_{\mathrm{S}}$ observed at low temperature is due to quasiparticles above the gap, or due to sub-gap transport, we have employed the additional voltage probes of sample $\mathrm{T} 2$ to characterize the point contacts. The local resistance $U_{\mathrm{A}} / I_{\mathrm{A}}$ of one contact is shown in Fig. 3 as a function of temperature. The data can be described by $R(T)=R_{0}+R_{\mathrm{BTK}}(T)+R_{\mathrm{ci}}(T)$, 


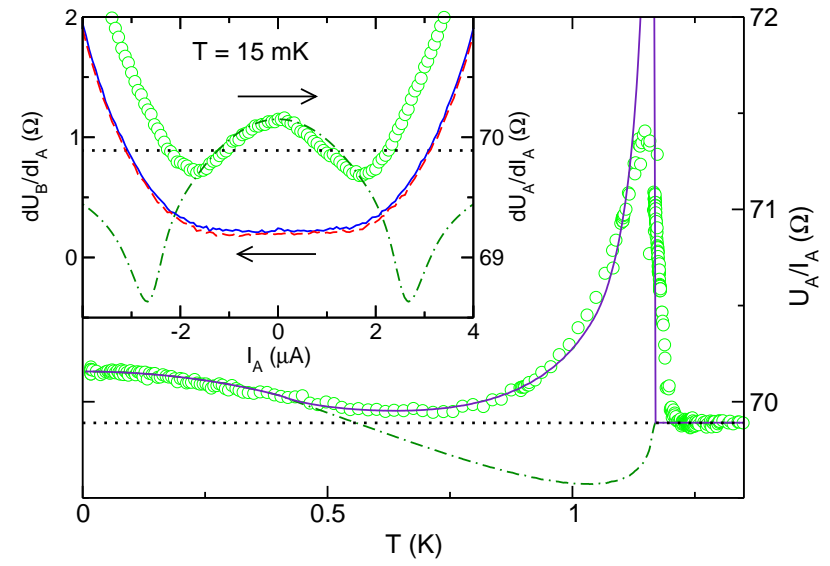

FIG. 3: (Color online) Symbols: Local resistance $U_{\mathrm{A}} / I_{\mathrm{A}}$ vs. temperature $T$ for a contact of sample T2. Solid line: fit to $R_{0}+R_{\mathrm{BTK}}(T)+R_{\mathrm{ci}}(T)$ (see text). Dash-dotted line: the contribution $R_{0}+R_{\mathrm{BTK}}(T)$.

Inset: Differential local resistance $d U_{\mathrm{A}} / d I_{\mathrm{A}}$ vs. $I_{\mathrm{A}}$ for the same contact (symbols), and differential non-local resistance $d U_{\mathrm{B}} / d I_{\mathrm{A}}$ for a detector at distance $d=210 \mathrm{~nm}$ (solid line: parallel, dashed line: antiparallel alignment). The dashdotted line is the fit $R_{0}+R_{\mathrm{BTK}}\left(U_{\mathrm{A}}\right)$ (see text), the dotted line indicates $R_{0}+R_{\mathrm{pc}}$ in both frames.

where $R_{0}=63.7 \Omega$ is the resistance of the ferromagnetic strip leading to the point-contact, $R_{\mathrm{BTK}}(T)$ is the temperature dependence of the point-contact resistance according to a spin-polarized extension of the BTK theory [14, 15], and $R_{\mathrm{ci}}(T)$ is a contact resistance due to charge imbalance 14, 16, 17, 18, which is essentially the same as observed in the non-local resistance. Parameters for the BTK model are $R_{\mathrm{pc}}=6.2 \Omega, P=0.45, Z=0.22$ and $\Delta=177 \mu \mathrm{eV}$ for the normal state point-contact resistance, the interfacial spin polarization, the interface transparency, and the gap, respectively. The fit to our model is excellent except for a slight broadening around $T_{\mathrm{c}}$.

The low bias part $\left(\left|I_{\mathrm{A}}\right|<1.7 \mu \mathrm{A}\right)$ of the differential local resistance $d U_{\mathrm{A}} / d I_{\mathrm{A}}$ can be well described by $R_{0}+R_{\mathrm{BTK}}\left(U_{\mathrm{A}}\right)$ using $U_{\mathrm{A}}=I_{\mathrm{A}}\left(R_{0}+R_{\mathrm{pc}}\right)$ (i.e. assuming insignificant inelastic scattering in the Fe strip) and the same parameters as for the temperature dependence, whereas the differential non-local resistance $d U_{\mathrm{B}} / d I_{\mathrm{A}}$ remains constant in that bias region (inset of Fig. 3). At higher bias $\left|I_{\mathrm{A}}\right|>1.7 \mu \mathrm{A}$, both local and non-local differential resistance show a steep increase due to charge imbalance, i.e. quasiparticle injection above the gap. The apparent reduction of the gap compared to the BTK prediction can be attributed to self-heating at higher bias. We conclude that the constant $\Delta R_{\mathrm{S}}$ observed in the lowbias region is due to sub-gap transport.

Sub-gap transport between normal metals and superconductors is mediated by Andreev reflection [19]: An electron from the normal metal enters the superconduc-

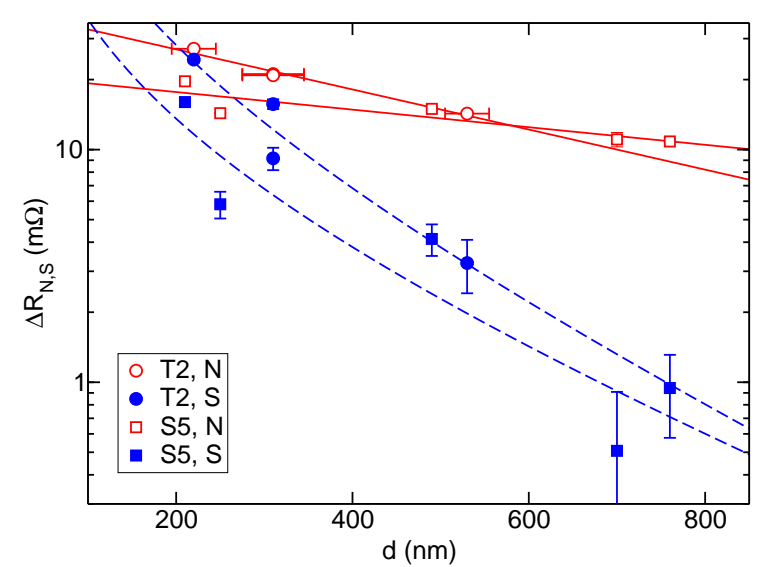

FIG. 4: (Color online) Difference $\Delta R_{\mathrm{N}, \mathrm{S}}$ between parallel and antiparallel alignment vs. distance $d$ between the contacts, for two samples in both the normal (open symbols) and superconducting (closed symbols) state. The solid and dashed lines are fits to (1) and (5), respectively, as described in the text.

tor, and a hole of opposite spin is retro-reflected, creating a spin-singlet Cooper pair in the superconductor. In a multi-terminal structure, it has been predicted that the incident electron and the retro-reflected hole may be transmitted through different contacts (crossed Andreev reflection, or CAR), as long as the distance between the two contacts does not exceed the superconducting coherence length [20, 21], yielding a negative non-local resistance. There is a second non-local process, electron cotunneling (EC), where an electron enters the superconductor through one contact, and an electron of the same spin leaves through the second contact, yielding positive non-local resistance. If the two contacts are spin polarized, EC and CAR are favorable for parallel and antiparallel magnetization alignment, respectively. We propose the superposition of CAR and EC processes as the origin of the observed non-local resistance difference in the superconducting state.

To obtain a quantitative description of our results, and to test our hypothesis, we have extracted $\Delta R_{\mathrm{N}}$ and $\Delta R_{\mathrm{S}}$ from the raw data and investigated their dependence on the distance $d$ between the injector and detector contact. These data are shown in Fig. [4 Both signals are of similar magnitude at short distance, and decay at increasing $d$. The decay in the superconducting state takes place on a much shorter length scale than in the normal state, indicating the different transport mechanism.

For the normal state data, we use a semiclassical model of spin injection and diffusion 22. The key parameters are the bulk spin polarization $p=\left(\sigma^{\mathrm{F} \uparrow}-\sigma^{\mathrm{F} \downarrow}\right) /\left(\sigma^{\mathrm{F} \uparrow}+\sigma^{\mathrm{F} \downarrow}\right)$ of the ferromagnet, where $\sigma^{\mathrm{F} \uparrow}$ and $\sigma^{\mathrm{F} \downarrow}$ are the spindependent conductivities of the ferromagnet, and the quantities $\mathcal{R}^{\mathrm{N}}=\rho^{\mathrm{N}} \lambda_{\mathrm{s}}^{\mathrm{N}} / A^{\mathrm{N}}$ and $\mathcal{R}^{\mathrm{F}}=\rho^{\mathrm{F}} \lambda_{\mathrm{s}}^{\mathrm{F}} / A^{\mathrm{J}}$, where $\rho^{(\mathrm{N}, \mathrm{F})}$ and $\lambda_{\mathrm{s}}^{(\mathrm{N}, \mathrm{F})}$ are the (spin-averaged) resistivity and 
spin-diffusion length in the normal metal and ferromagnet, respectively, $A^{\mathrm{N}}$ is the cross section of the normal metal and $A^{\mathrm{J}}$ is the contact area. For our samples, we estimate $\left(\mathcal{R}^{\mathrm{F}} \approx 3 \Omega\right)>\left(\mathcal{R}^{\mathrm{N}} \approx 1 \Omega\right)$. In that limit, and neglecting contact resistances and inhomogenous current distributions, $\Delta R_{\mathrm{N}}$ is given by

$$
\Delta R_{\mathrm{N}} \approx p^{2} \frac{\rho^{\mathrm{N}} \lambda_{\mathrm{s}}^{\mathrm{N}}}{A^{\mathrm{N}}} \exp \left(-d / \lambda_{\mathrm{s}}^{\mathrm{N}}\right) .
$$

A joint description of both local and non-local sub-gap conductance in the superconducting state is given by 23 .

$$
\left(\begin{array}{c}
I_{\mathrm{A}} \\
I_{\mathrm{B}}
\end{array}\right)=\left(\begin{array}{cc}
G_{\mathrm{A}} & G_{\mathrm{CAR}}-G_{\mathrm{EC}} \\
G_{\mathrm{CAR}}-G_{\mathrm{EC}} & G_{\mathrm{B}}
\end{array}\right)\left(\begin{array}{c}
U_{\mathrm{A}} \\
U_{\mathrm{B}}
\end{array}\right),
$$

where $I_{\mathrm{A}, \mathrm{B}}, U_{\mathrm{A}, \mathrm{B}}$ and $G_{\mathrm{A}, \mathrm{B}}$ are the current, voltage and local conductance of the injector (A) and detector (B) contact, $G_{\mathrm{CAR}}$ and $G_{\mathrm{EC}}$ are the non-local conductances due to CAR and EC processes, and using $G_{\mathrm{A}}, G_{\mathrm{B}} \gg$ $G_{\mathrm{CAR}}, G_{\mathrm{EC}}$ throughout. Inverting (2) for the case $I_{\mathrm{B}}=0$ (voltage detection at $\mathrm{B}$ ) we find the non-local resistance

$$
R_{\mathrm{S}}=U_{\mathrm{B}} / I_{\mathrm{A}} \approx-\left(G_{\mathrm{CAR}}-G_{\mathrm{EC}}\right) /\left(G_{\mathrm{A}} G_{\mathrm{B}}\right) .
$$

Quantitative predictions for a diffusive superconductor yield, to lowest order in transmission coefficients [24]

$$
\left(\begin{array}{c}
G_{\mathrm{CAR}} \\
G_{\mathrm{EC}}
\end{array}\right) \approx \frac{\pi}{4}\left(\begin{array}{c}
G_{\mathrm{A}}^{\uparrow} G_{\mathrm{B}}^{\downarrow}+G_{\mathrm{A}}^{\downarrow} G_{\mathrm{B}}^{\uparrow} \\
G_{\mathrm{A}}^{\uparrow} G_{\mathrm{B}}^{\uparrow}+G_{\mathrm{A}}^{\downarrow} G_{\mathrm{B}}^{\downarrow}
\end{array}\right) \frac{\exp (-d / \xi)}{N e^{2} D d},
$$

where $G_{\mathrm{A}, \mathrm{B}}^{\uparrow, \downarrow}$ are the spin-dependent contact conductances, and $N, D$ and $\xi$ are the density of states at the Fermi level, the diffusion coefficient and the dirty limit coherence length of aluminum, respectively. Using Einstein's relation $N e^{2} D=1 / \rho^{\mathrm{N}}$, we find $R_{\mathrm{S}} \approx$ $\pm P_{\mathrm{A}} P_{\mathrm{B}} \pi \rho^{\mathrm{N}} \exp (-d / \xi) / 4 d$, where + and - are for parallel and antiparallel configuration, and $P_{\mathrm{A}, \mathrm{B}}=\left(G_{\mathrm{A}, \mathrm{B}}^{\uparrow}-\right.$ $\left.G_{\mathrm{A}, \mathrm{B}}^{\downarrow}\right) / G_{\mathrm{A}, \mathrm{B}}$ are interfacial spin polarizations, and with $P=P_{\mathrm{A}}=P_{\mathrm{B}}$

$$
\Delta R_{\mathrm{S}} \approx P^{2} \frac{\pi}{2} \rho^{\mathrm{N}} \frac{\exp (-d / \xi)}{d} .
$$

Recent predictions for high transparency interfaces indicate the same distance dependence and an amplitude within a factor of three of the low-transparency case 25].

Fits of (11) and (5) to our data in the normal and su-

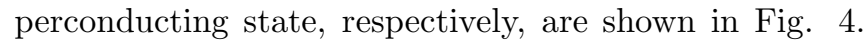
Within the experimental scatter, the data are described well by the fits. We find $\lambda_{\mathrm{S}}^{\mathrm{N}}=500-1000 \mathrm{~nm}$, consistent with values for aluminum obtained by spin-valve experiments [26], and a bulk spin polarization $p \approx 20 \%$. The interfacial spin polarization $P \approx 50 \%$ obtained from the fits in the superconducting state is in excellent agreement with $P \approx 45 \%$ obtained from point contact spectroscopy on iron [3, 15], and $\xi \approx 300 \mathrm{~nm}$ is consistent with the dirty limit predictions calculated from the resistivity. Thus, our data in the superconducting state are in good quantitative agreement with the predicted behavior.

To conclude, we have investigated experimentally nonlocal sub-gap transport in superconductors-ferromagnet hybrid structures, and shown evidence for the superposition of crossed Andreev reflection (CAR) and electron cotunneling. CAR has been discussed as a sensitive probe of superconducting order parameters 20]. In our case of a dirty (hence isotropic) BCS superconductor, the order parameter enters only through an exponential decay with $\xi$. With sufficient spatial resolution an investigation of unconventional superconductors appears feasible. More recent proposals in the context of quantum information processing (see 27] and references therein) have pointed out that CAR, if viewed as the decay of a Cooper pair into two electrons in different normal metal leads, creates a spatially separated entangled electron pair. This entanglement can be probed by measuring noise crosscorrelations in our structures.

Useful discussions with B. J. van Wees, C. Strunk and M. Aprili are gratefully acknowledged. This work was supported by the Deutsche Forschungsgemeinschaft within the framework of the Center for Functional Nanostructures.

* e-mail address: detlef.beckmann@int.fzk.de

[1] M. A. Sillanpää, T. T. Heikkilä, R. K. Lindell, and P. J. Hakonen, Europhys. Lett. 56, 590 (2001).

[2] T. Kontos, M. Aprili, J. Lesueur, and X. Grison, Phys. Rev. Lett. 86, 304 (2001).

[3] R. J. J. Soulen, J. M. Byers, M. S. Osofsky, B. Nadgorny, T. Ambrose, S. F. Cheng, P. R. Broussard, C. T. Tanaka, J. Nowak, J. S. Moodera, et al., Science 282, 85 (1998).

[4] S. K. Upadhyay, A. Palanisami, R. N. Louie, and R. A. Buhrman, Phys. Rev. Lett. 81, 3247 (1998).

[5] F. Pérez-Willard, J. C. Cuevas, C. Sürgers, P. Pfundstein, J. Kopu, M. Eschrig, and H. v. Löhneysen, Phys. Rev. B 69, 140502 (2004).

[6] J. Y. Gu, J. A. Caballero, R. D. Slater, R. Loloee, and W. P. Pratt Jr., Phys. Rev. B 66, 140507 (2002).

[7] V. V. Ryazanov, V. A. Oboznov, A. Y. Rusanov, A. V. Veretennikov, A. A. Golubov, and J. Aarts, Phys. Rev. Lett. 86, 2427 (2001).

[8] T. Kontos, M. Aprili, J. Lesueur, F. Genet, B. Stephanidis, and R. Boursier, Phys. Rev. Lett. 89, 137007 (2002).

[9] A. Bauer, J. Bentner, M. Aprili, M. L. Della Rocca, M. Reinwald, W. Wegscheider, and C. Strunk, Phys. Rev. Lett. 92, 217001 (2004).

[10] M. Johnson and R. H. Silsbee, Phys. Rev. Lett. 55, 1790 (1985).

[11] J. Clarke, Phys. Rev. Lett. 28, 1363 (1972).

[12] M. Tinkham and J. Clarke, Phys. Rev. Lett. 28, 1366 (1972).

[13] M. Johnson, Appl. Phys. Lett. 65, 1460 (1994).

[14] G. E. Blonder, M. Tinkham, and T. M. Klapwijk, Phys. 
Rev. B 25, 4515 (1982).

[15] G. J. Strijkers, Y. Ji, F. Y. Yang, C. L. Chien, and J. M. Byers, Phys. Rev. B 63, 104510 (2001).

[16] C. C. Chi and J. Clarke, Phys. Rev. B 19, 4495 (1979).

[17] C. C. Chi and J. Clarke, Phys. Rev. B 21, 333 (1980).

[18] T. Y. Hsiang and J. Clarke, Phys. Rev. B 21, 945 (1980).

[19] A. F. Andreev, Sov. Phys. JETP 19, 1228 (1964).

[20] J. M. Byers and M. E. Flatté, Phys. Rev. Lett. 74, 306 (1995).

[21] G. Deutscher and D. Feinberg, Appl. Phys. Lett. 76, 487 (2000).
[22] S. Takahashi and S. Maekawa, Phys. Rev. B 67, 052409 (2003).

[23] G. Falci, D. Feinberg, and F. W. J. Hekking, Europhys. Lett. 54, 255 (2001).

[24] D. Feinberg, Eur. Phys. J. B 36, 419 (2003).

[25] R. Mélin and D. Feinberg (2004), cond-mat/0407283.

[26] F. J. Jedema, M. S. Nijboer, A. T. Filip, and B. J. van Wees, Phys. Rev. B 67, 085319 (2003).

[27] P. Recher and D. Loss, Phys. Rev. Lett. 91, 267003 (2003). 